\title{
El Flamenco Americano (Phoenicopterus ruber) en la Orinoquia Colombiana: ¿Ampliación de Rango o introducción de Especie?
}

\author{
The American flamingo (Phoenicopterus ruber) in the Colombian \\ Orinoco region: extending its range or the introduction of the species?
}

\section{O Flamingo-americano (Phoenicopterus ruber) na Orinoquía colombiana: ampliação da sua distribuição geográfica ou introdução da espécie?}

Johanna I. Murillo - Pacheco ${ }^{1 *}$,Wilian F. Bonilla - Rojas ${ }^{2}$, Germán López-Iborra ${ }^{3}$

BSc, PhD, Centro Iberoamericano de la Biodiversidad (CIBIO), Departamento de Ciencias Ambientales y Recursos Naturales, Universidad de Alicante, Alicante, España. Campus San Vicente del Raspeig s/n, Apdo. 99, E-03080.

BSc,Corporación Llanera de Ornitología y de la Naturaleza - KOTSALA, Villavicencio Colombia.

BSc, PhD, Instituto Ramón Margalef IMEM, Departamento de Ecología, Universidad de Alicante, Alicante, España.

Grupo de Investigación Biorinoquia, Facultad Ciencias Básicas y Ecología, Universidad de los Llanos, Villavicencio, Colombia.

Email: johannamurillo@gmail.com

Recibido: Febrero 13 de 2013 Aceptado: Diciembre 18 de 2013

\section{Resumen}

Se presentan los primeros registros del flamenco americano (Phoenicopterus ruber) para el piedemonte del departamento del Meta en la Orinoquia Colombiana. Se realizaron tres observaciones en vida silvestre de la especie en las zonas inundadas de un cultivo de arroz de riego (Oryza sativa) en los años 2008 y 2010. Estos registros podrían corresponder a la ampliación de rango de la especie, a la presencia de individuos vagabundos o a la introducción tipo trasplante de la especie a esta región del país. Se analizó la situación de $P$. ruber en la Orinoquia Colombiana, se proponen las posibles rutas de arribo de la especie a esta región del país (si fuese una ampliación de rango geográfico) y se discuten la tenencia de fauna silvestre en cautiverio.

Palabras clave: Llanos orientales, introducción, tráfico ilegal fauna, arrozales, Oryza sativa

\begin{abstract}
The first sightings of the American or Caribbean flamingo (Phoenicopterus ruber) in the foothills of the Meta department in the Colombian Orinoco region are presented here. Three observations of the species were made in the wild in the flooded areas of an irrigated rice (Oryza sativa) paddy from 2008 to 2010. These records could show how the species' range is becoming extended, the presence of individual vagrants or the specie's transplant-type introduction into this region of Colombia. The circunstances regarding $P$. ruber's presence in the Colombian Orinoco region is analysed, possible arrival routes for the species to this region of Colombia are proposed (if one is dealing with an expansion of geographical range) and captive wildlife occupancy are discussed.
\end{abstract}

Key words: Colombian Eastern plains, introduction, illegal wildlife trade, rice paddy, Oryza sativa. 


\section{Resumo}

O trabalho apresenta os primeiros registros do Flamingo-americano (Phoenicopterus ruber) para a região da base da montanha, do departamento de Meta em Orinoquia Colombiana. Foram realizadas três observações sobre a vida silvestre desta espécie em regiões com cultivo de arroz alagado (Oryza sativa) durante os anos de 2008 e 2010. Esses registros demonstram o crescente leque de espécies, e a presença de indivíduos que perderam ás áreas para sobreviver, representando a transposição desta espécie para esta área do país. A situação analisada em $P$. Ruber na Orinoquia Colombiana propõe a existência de possíveis rotas que permitem a chegada desta espécie a essa região, e discutem as implicações da tendência de manutenção da fauna silvestre em cativeiro.

Palavras-chave: planícies do leste, introdução, tráfico de animais selvagens, arroz, Oryza sativa

\section{Introducción}

El flamenco caribeño o americano Phoenicopterus ruber, habita desde el nivel del mar hasta los 200 msnm(Hilty y Brown, 2001), en cuerpos de agua poco profundos dulces y salobres. En Colombia la distribución natural de la especie abarca las costas de los departamentos de la Guajira y Magdalena (Troncoso, 2002), aunque cuenta con registros en el interior del país en los departamentos de Caldas (CENICAFE, 2006), Valle del Cauca (Cifuentes y Castillo, 2009; 2010), Risaralda (CARDER, 2011) y en el pacífica (Ruizet al. 2007; Isaza, Comunicación, personal 2011) que corresponden probablemente a individuos erráticos o fugados.

En la región de la Orinoquia la especie se ha registrado en los Llanos de Venezuela, en los Hatos El Cedral (Pérez, J., Comunicación personal 2008) y El Frio (McNish, 2007), en el Estado de Apure. Mientras para Colombia se conoce un registro en las cercanías del caño El Tigre en el río Cravo sur en el departamento de Casanare (Mejía-Tobon, A., Comunicación. personal 2008) con procedencia desconocida y por último se cuenta con las observaciones realizadas en el piedemonte del departamento del Meta en los años 2008 y 2010.

A través de los nuevos registros de $P$. ruberen el Meta aportados en este documento, se cuestiona el origen de los individuos y se discute sobre la posibilidad de ampliación de rango de la especie a esta región a través de diferentes rutas de origen, a individuos errantes o a la introducción de la especie (tipo trasplante) en la región de la Orinoquia Colombiana. Las aves pueden realizar grandes desplazamientos especialmente migratorios desde transfronterizos o latitudinales, hasta locales y altitudinales (Fierro, 2009), también pueden desplazarse de forma errática de acuerdo a las condiciones de tiempo y recursos. Particularmente el $P$. ruber es considerado en Colombia un migrante local que realiza movimientos con una temporalidad estacional, de forma longitudinal y transfronteriza (Rodríguez y Pantaleón, 2012).

A nivel internacional el Flamenco caribeño se encuentra en la categoría II del Apéndice Cites y según la UICN es una especie de Preocupación Menor (LC) (BirdLife International, 2012; CITES, 2013). Sin embargo es de resaltar que en Colombia es una especie Vulnerable (VUB1ab (i,iii) C2a (ii)) principalmente por la pérdida del hábitat y a la presión proveniente del tráfico ilegal (Renjifo et al., 2002; Troncoso, 2002) hasta la desaparición de poblaciones locales (Rueda, 1999). Ha sido introducida en el interior del país, como ave de ornamento y su introducción corresponde al tipo de especies trasplantadas, las cuales tienen por origen el mismo país, pero son trasladadas de una región natural a otra ó entre cuencas biogeografías (Baptistey Múnera, 2010).

Es conocido que la introducción de especies trae consigo implicaciones ecológicas y ambientales (Manchester y Bullock, 2000; McNeely et al., 2001; Mock et al., 2004; Baptiste et al., 2010), incrementadas aún más cuando los individuos son liberados al medio natural. Una de la principales causas de la introducción de especies es el tráfico ilegal, considerada una de las actividades ilícitas más lucrativas, después del comercio de drogas y armas a nivel global (Roe, 2008). En Colombia es escasa la información, se basa casi que exclusivamente a registros de decomisos (MMA, 2002; Mancera y Reyes, 2008).

En la Orinoquia Colombiana sobresale la comercialización y tenencia como mascotas de animales ornamentales (Aguilar, 2004), siendo las aves uno de los grupos preferidos. Por tanto, se hace necesario tener un mayor control sobre estas actividades, evitar los permisos de introducción e implementar estrategias de educación y concienciación. Así como también, em- 
prender acciones de gestión de los individuos exóticos que ya se encuentran en la Orinoquia Colombia, especialmente la reubicación a sus hábitats naturales conocidos, teniendo en cuenta las implicaciones que pude generar sobre las poblaciones nativas la liberación de animales que han sido confinados (Jiménez y Cadena, 2004).

\section{Materiales y métodos}

\section{Área de estudio}

Los registros visuales se llevaron a cabo en el corregimiento de Pachaquiaro en el municipio de Puerto López, departamento del Meta, específicamente en la Hacienda Arrocera Providencia ubicada en el kilometro 56 en la vía que conduce de Villavicencio a Puerto López (44'2.58" N, 738’12.84" O, 197 msnm) (Figura 1). Esta zona hace parte del piedemonte bajo de la cordillera Oriental de los Andes. Se encuentra en el ecosistema denominado Zonobioma húmedo tropical (ZHT) piedemonte del Meta (Romero et al., 2004), el clima es muy húmedo tropical cálido, con una temperatura media anual de $25{ }^{\circ} \mathrm{C}$ y la precipitación anual entre 1,500 y 3,500 mm (Rangel y Aguilar, 1995; Rangel, 1998). El uso principal del suelo en esta zona es el agropecuario con cultivo de arroz y palma, ganadería vacuna y la exploración y extracción petrolera.

\section{Muestreo}

Se realizaron visitas al área de estudio entre los años 2008 y 2010 de acuerdo al desarrollo de los proyectos de investigación de la Corporación Llanera de Ornitología y de la Naturaleza - Kotsala. Se Ilevaron a cabo 25 salidas de campo a la zona, en las cuales se monitoreo la avifauna y se hizo búsqueda visual del flamenco americano sobre rutas establecidas que incluyeron senderos, carreteras y lotes del cultivo de arroz en diferentes estados de desarrollo. Se emplearon binoculares 10X 42 y en algunas ocasiones telescopio 20-60X $80 \mathrm{~mm}$.

\section{Recopilación de información}

Se llevó a cabo una revisión de información bibliográfica disponible, la consulta a ornitólogos y observadores de aves. Por último, se realizó una solicitud formal a la entidad ambiental competente: la Corporación

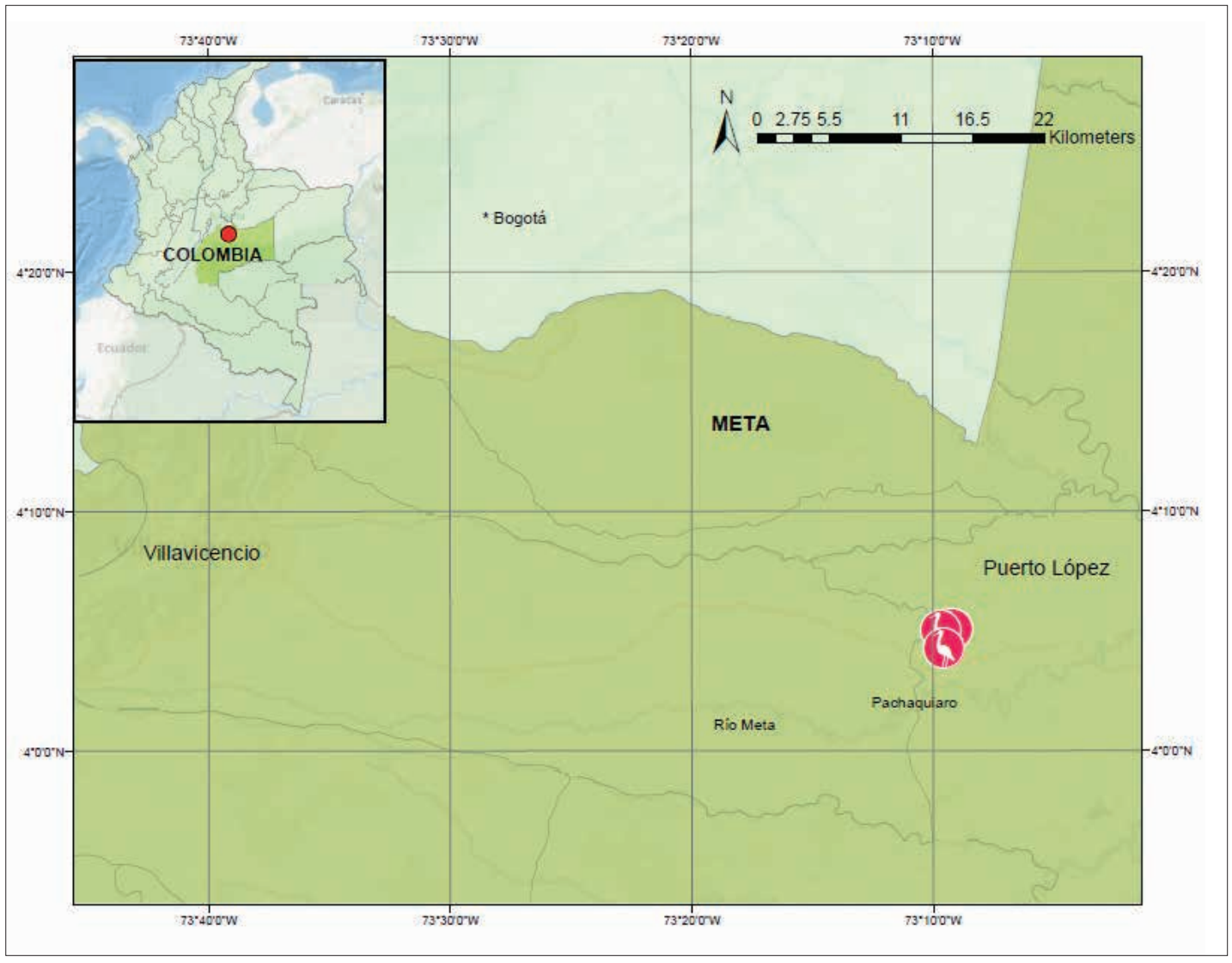

Figura 1. Localidad de observación del flamenco americano (P. ruber) en la Hacienda Arrocera Providencia, corregimiento de Pachaquiaro, Puerto López - Meta, Colombia. 
Figura 2. Individuos de Flamenco americano (P. ruber) observados en la Hacienda Arrocera Providencia, Puerto López - Meta (Colombia). A: En un lote de arroz de aproximadamente 25 días de sembrado (10-Ene-2008). B: Lote de arroz de $10 \mathrm{~cm}$ de altura aproximadamente (19-Jun-2010).

para el desarrollo sostenible del área de manejo especial La Macarena - CORMACARENA en el año 2010 de los registros de esta especie en el departamento del Meta, correspondientes a decomisos, permiso de tenencia y núcleos reubicadores.

\section{Resultados}

El flamenco americano (Phoenicopterus ruber) se observó en tres ocasiones en las zonas inundadas de un cultivo de arroz en el piedemonte bajo del departamento del Meta. La primera observación se realizó el 10 de Enero de 2008 (Murillo J, Bonilla WF, Millan C), en un terreno inundado en un lote de arroz de aproximadamente 25 días de sembrado. El individuo tenía una coloración rosa muy tenue (Figura 2), se encontraba en una laguna temporal y forrajeaba entre el agua y el lodo en compañía de otras aves acuáticas como gallitos de ciénaga (Jacana jacana), patos caretos (Dendrocygna viduata), patos sirirí (Dendrocygna autumnalis) y el Andarrío solitario (Tringa solitaria).

El segundo registro fue el 31 de Agosto de 2008 (Millán C), en una charca con $20 \mathrm{~cm}$ de profundidad, en un campo de arroz con riego y 40 días de siembra aproximadamente. El Flamenco compartía hábitat y forrajeaba con gallitos de ciénaga (J. jacana) y cigüeñuelas (Himantopus mexicanus). La tercera observación fue el 19 de Junio de 2010 (Murillo J, Bonilla WF), en un lote de arroz inundando de $10 \mathrm{~cm}$ de altura y 10 días de sembrado aproximadamente. El individuo sobrevolaba el área y forrajeaba cerca de un canal de riego interno y una laguna de inundación, además presentaba una coloración más oscura y uniforme respecto a los registros anteriores, y se encontraba junto a patos caretos (D. viduata) y patos sirirí (D. autumnalis) (Figura 3).

\section{Información secundaria}

Se encontraron registros disponibles del Flamenco americano para la Orinoquia de Colombia, en los departamentos de Casanare y Meta. En Mayo de 2008 se registró en Casanare, en las cercanías del caño El Tigre en el río Cravo sur (Mejía-Tobón, A., Comunicacion personsl 2008). Para el Meta se obtuvieron exclusivamente registros de animales en cautiverio y decomisados por la Corporación para el desarrollo sostenible del área de manejo especial La Macarena
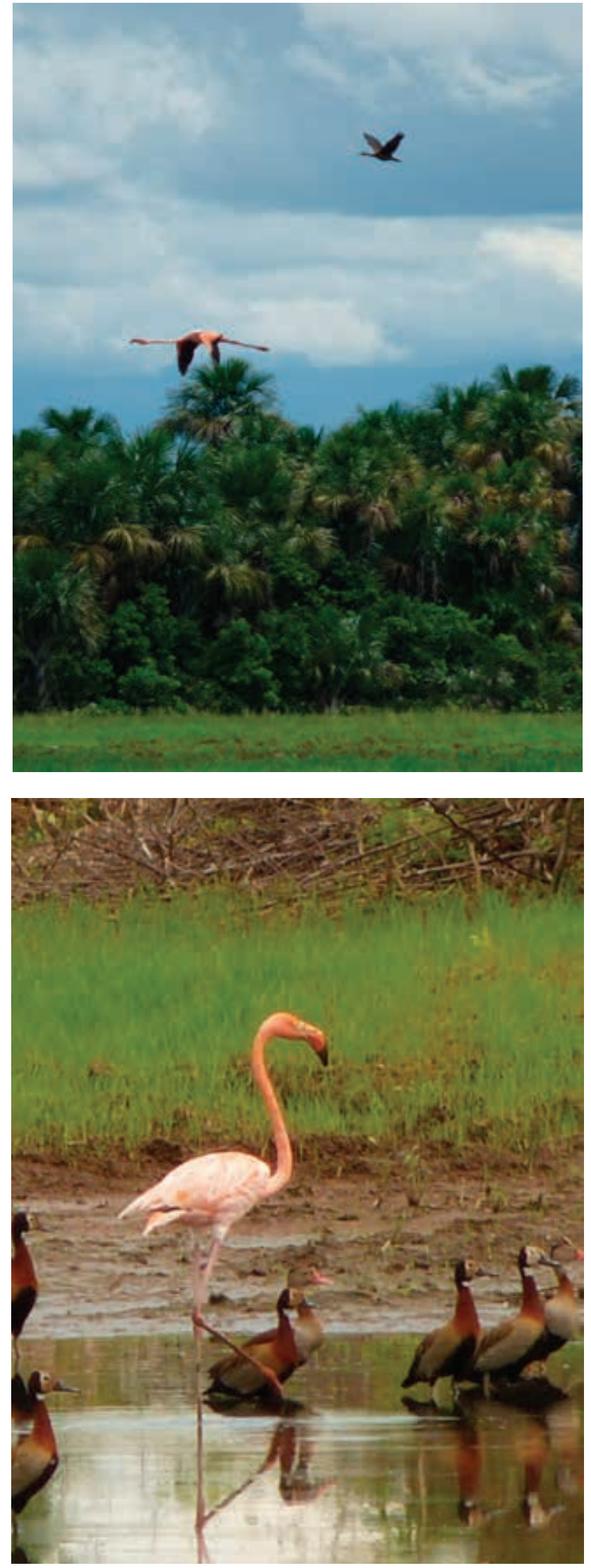

Figura 3: Flamingo 
- Cormacarena. Entidad que informó sobre tener conocimiento y reporte de 14 individuos de ésta especie, cuatro individuos en el Hotel La Macarena en la vereda El Choapal del Municipio de Restrepo; cinco individuos en el Hotel Santa Barbará en el municipio de Villavicencio, que fueron reubicados en el aviario nacional de Colombia en Isla Barú en Cartagena.

Se registran adicionalmente, registran tres individuos en la Quinta el Diamante en la vereda La Unión del municipio de Villavicencio y reportan el decomiso de dos individuos en el terminal de transportes de Villavicencio, los cuales fallecieron durante el proceso de valoración (Cormacarena, 2010). Posteriormente, a través de visitas por algunas de las localidades reportadas y a la consulta con pobladores locales, se encontraron dos individuos adicionales en la finca Manigua en la vereda la Llanerita y se registró la fuga de dos individuos más en el Centro Vacacional los Gavanes en la vereda Murujuy, ambas localidades en el municipio de Villavicencio y no incluidas en los registros de la entidad ambiental Cormacarena.

\section{Discusión}

El interés de los tres registros en vida silvestre de $P$. ruber realizados en el departamento del Meta, cuestiona si los reportes corresponden a la ampliación de la distribución geográfica de la especie, si son solo individuos vagabundos con movimientos fuera de su rango o si por el contrario son individuos fugados producto de la introducción de la especie a esta región. El interrogante radica en que dichos registros se encontraron en una localidad por fuera del rango de distribución natural conocida para la especie en Colombia (Hilty y Brown, 2001; Troncoso, 2002; Birdlife International, 2012).

Es importante resaltar que la especie tiene una amplia distribución con una extensión de presencia de aproximadamente $20.000 \mathrm{~km}^{2}$, con registros de individuos vagabundos en más de diez países incluido los más lejanos como Canadá y Estados Unidos (Birdlife International, 2012). Por tanto, se asume la capacidad que tiene la especie de recorrer largas distancias y realizar grandes desplazamientos. Posee un tamaño poblacional fluctuante y se estiman entre 50.000 a 60.000 individuos en movimiento en la península de la Guajira en Colombia, la costa de Venezuela y las islas cercanas, incluyendo Bonaire (Espinoza et al., 2000; Rodríguez y Pantaleón, 2012).

En el caso que fueran movimientos de la especie, se sugieren como posibles orígenes, cinco zonas: 1. Costa Caribe Venezolana, 2. Orinoquia Venezolana, 3.
Caribe Colombiano, 4. Andes Colombianos y 5. Costa pacífica. Los individuos observados podrían provenir de la costa Caribe de Venezuela donde se estima una población aproximada de 37.110 individuos, correspondientes al 38\% de toda la población de la especie (Espinoza et al., 2000), por consiguiente tendrían que recorrer entre 700 a 900 kilómetros lineales y atravesar diferentes ecosistemas. Otra ruta que podrían utilizar seria el río Orinoco desde el Delta, aunque la porción del Delta del Orinoco es considerado por Espinoza et al., (2000), como un hábitat pobre de presencia de esta especie y desde allí los individuos tendrían que recorrer más de 1440 kilómetros lineales hasta el Corregimiento de Pachaquiaro en Colombia. Por consiguiente es una ruta bastante larga y riesgosa.

La otra ruta desde Venezuela seria las tierras de los Ilanos de la Orinoquia desde el estado de Apure, donde se han reportado algunos individuos (McNish, 2007; Pérez J., Comunicación personal 2008), en los hatos El Cedral y el Frio. Esta zona dista a menos de 600 kilómetros lineales y corresponde a la misma unidad biogeografía y ecosistemas que la localidad de estudio. Por ello, este podría ser el origen silvestre más acertado de procedencia de los registros del Meta, pero los reportes mencionados de Venezuela, son individuos aislados y no una población establecida, lo cual indica que es poco probable el desplazamiento de individuos entre las dos zonas Orinocenses en ambos países.

Si la procedencia de los individuos fuera Colombia, el área de mayor probabilidad seria el Caribe y específicamente la península de la Guajira, donde se encuentra la mayor concentración de la especie en el país. Se ha estimado la población en 5818 individuos aproximadamente (Troncoso, 2002) y datos más puntuales proponen rangos entre ocho y 3000 individuos en el departamento de la Guajira (Ruiz et al., 2008). Si la especie se desplazara desde esta zona, los individuos tendrían un recorrido de alrededor $900 \mathrm{~km}$ lineales y tendrían que atravesar la cordillera Oriental de los Andes, evento poco factible ya que esta cordillera actúa como barrera efectiva en la dispersión de especies, aunque se cuenta con registros en la ciénaga de zapatos como extremo sur en su distribución natural en el país (Hilty y Brown, 2001).

La cuarta zona probable sería el interior de Colombia en la región Andina, en donde la especie se ha registrado con anterioridad en algunas localidades fuera de su distribución natural, la mayoría corresponden a observaciones esporádicas (Cenicafe, 2006; Cifuentes y Castillo, 2009; 2010). Dos individuos se observaron en la Represa de Cameguadua, Departamento de Caldas, donde se sugiere que pueden tratarse de individuos 
que viajaron a lo largo del valle del Río Cauca y posteriormente por los Ríos Guacaica y Chinchiná. Así mismo, podría deberse a individuos fugados de un zoológico (Cenicafe, 2006). Entre 2008 y 2009 se registraron tres individuos en La Laguna de Sonso, departamento del Valle del Cauca, los cuales son considerados como introducidos en la zona (Cifuentes y Castillo, 2009; 2010). Además la Corporación Autónoma Regional de Risaralda (CARDER, 2011), reportó la incautación de 12 individuos en la vereda Malabar en el Municipio de Pereira en Mayo de 2011.

La quinta y última opción considerada es la costa Pacífica, con el registro de un individuo en 1998 (El cual tiene duda en su identificación por que podría ser Phoenicopterus chilensis) y dos individuos más confirmados de P. ruber en 2003, el origen de estos podría ser fugas de cautiverio o al movimiento de organismos procedentes de la costa ecuatoriana (Ruiz et al., 2007). También se cuenta con el reporte de una pareja en el río valle, en Bahía Solano - Chocó (Isaza C., Comunicación personal 2011).Esta es la opción con menor posibilidad de ser el origen de los individuos observados en la Orinoquia Colombiana, debido a que estos tendrían que atravesar las tres cordilleras de los Andes para llegar al departamento del Meta.

Para la zona de la Orinoquia Colombiana, el único registro en vida silvestre conocido es en el piedemonte del departamento del Casanare (Mejía-Tobón A., Comunicación personal 2008), pero al carecer de información adicional no permite su relación con los registros del departamento del Meta, debido a que podría tratarse de un individuo errático, fuga de cautiverio, o aunque, también podría haber arribado a los Ilanos a través de alguna de las rutas planteadas anteriormente.

Respecto a la posibilidad que la especie se encuentre en estado silvestre en los Llanos orientales colombianos como consecuencia de un evento de trasplante de la especie, de momento no es concluyente. Aunque se carece de información referente suficiente para confirmarlo, se tiene el reporte de 12 individuos en cautiverio para el departamento del Meta (CORMACARENA, 2010), y las observaciones ya referenciadas anteriormente en este documento. Por ello, a $P$. ruber se lo podría considerar como una especie de ornato exótica en la Orinoquía, de la cual se han reportado fugas ocasionales al medio silvestre desde sus sitios de confinamiento en fincas de recreo y hoteles.

Todas las localidades reportadas por la entidad ambiental CORMACARENA se encuentran a más de 50 kilómetros del sitio de observación de los individuos. Sin embargo, los pobladores locales del corregimiento de
Pachaquiaro informaron la tenencia de esta especie en el Centro Vacacional los Gavanes, donde sus propietarios confirmaron la fuga de dos individuos que tenían en cautiverio en 2009. La finca turística Los Gavanes se ubica aproximadamente a 13 kilómetros en línea recta de la Hacienda arrocera Providencia, donde fue observado el Flamenco americano. Esta cercanía sugiere una amplia posibilidad de movimiento de los individuos después de la fuga y una alta probabilidad que correspondan a ellos. No es posible la afirmación de este supuesto debido a que los especímenes en tenencia no cuentan con ningún tipo de marcaje como anillos de colores o marcas alares que permita su reconocimiento.

Dado lo anterior, se hace necesario ampliar el esfuerzo de control y vigilancia de la tenencia de fauna de silvestre en esta región del país, que permita implementar mejores opciones de reubicación de individuos exóticos, aunque el retorno de estos individuos a sus zonas de distribución natural, puede conllevar implicaciones sanitarias y biológicas (Jiménez y Cadena, 2004). Por ello, es preciso emprender acciones conjuntas de investigación con Universidades, ONG y diferentes entidades que puedan integrar sus capacidades, así como la articulación de esfuerzos con las entidades competentes ambientales y judiciales. Se debe ejercer mayor seguimiento a aquellos individuos que se conozca su tenencia y que sean de difícil reubicación. Así mismo, implementar métodos efectivos de marcaje que permitan su posible seguimiento en caso de fuga, y como eje transversal incluir la temática en las estrategias de educación ambiental a todos los niveles de la población y se sugiere ampliar la búsqueda del flamenco americano en la región.

\section{Agradecimientos}

Los autores agradecen a la Corporación Llanera de Ornitología y de la Naturaleza - Kotsala, al Fondo Humedales para el Futuro (FHF) de la Convención Ramsar sobre los Humedales (Proyecto WFF/09/CO/5), al fondo de formación doctoral en el exterior (COLCIENCIAS - Convocatoria 512 de 2010), al programa Doctorado 2008 de la Fundación Carolina y Colfuturo. También ofrecen un especial agradecimiento a Carlos Millán por compartir su interés por las aves asociadas al agroecosistema del arroz y por su registro. A Jorge Pérez (Apure), Alejandro Mejía Tobon (Casanare) y Cesar Isaza (Choco) por facilitar sus observaciones personales de la especie. También a Andrea Monroy, Ricardo Murillo, Adolf Ciouderis, Santiago Pérez y Laura Oyola por su compañía y apoyo en diferentes salidas de campo. A Thomas McNish por todo lo enseñado sobre las aves de los Llanos. En especial queremos agradecen a los revisores del manuscrito. 


\section{Referencias}

Aguilar C. 2004. Diagnóstico de la fauna decomisada en las jurisdicciones de CORPORINOQUía y CormaCARENA. Instituto de Investigación de Recursos Biológicos Alexander von Humboldt - IAvH. Programa de Uso y Valoración de la Biodiversidad. Bogotá, Colombia, p 276.

Baptiste MP, Castaño N, Cárdenas D, Gutiérrez FP, Gil DL, Lasso CA (Editores). 2010. Análisis de riesgo y propuesta de categorización de especies introducidas para Colombia. Instituto de Investigación de Recursos Biológicos Alexander von Humboldt - IAvH. Bogotá, Colombia, p. 200.

Baptiste MP, Múnera C. 2010. Análisis de riesgo de vertebrados terrestres introducidos en Colombia. En: Baptiste MP, Castaño N, Cárdenas D, Gutiérrez FP, Gil DL, Lasso CA (Editores). 2010. Análisis de riesgo y propuesta de categorización de especies introducidas para Colombia. Instituto de Investigación de Recursos Biológicos Alexander von Humboldt - IAvH. Bogotá, Colombia, p. 149-199.

BirdLife International. 2012. Phoenicopterus ruber. In: IUCN 2012. IUCN Red List of Threatened Species. Version 2013.2. <www.iucnredlist.org>. Downloaded on 13 February 2014.

CARDER - Corporación Autónoma Regional de Risaralda. 2011. Auto de inicio de investigación 0266. Pereira, Colombia.

CENICAFE - Centro Nacional de Investigaciones del Café. 2006. Un flamenco en la represa de Cameguadua en Chinchiná. Biocarta Marzo 10 de 2006. Chinchina, Colombia.

Cifuentes-Sarmiento Y, Castillo-Cortés LF. 2009. Colombia: informe anual. Censo Neotropical de Aves Acuáticas 2008 [en línea]. En: Unterkofler DA, Blanco DE. (Editores): El Censo Neotropical de Aves Acuáticas 2008; Una herramienta para la conservación. Wetlands International, Buenos Aires, Argentina http://lac. wetlands.org/

Cifuentes-Sarmiento Y, Castillo-Cortés LF. 2010. Colombia: Informe Anual. Censo Neotropical de Aves Acuáticas 2009 [en línea]. En: Unterkofler DA, Blanco DE. (Editores): El Censo Neotropical de Aves Acuáticas 2009. Wetlands International, Buenos Aires, Argentina http:// lac.wetlands.org/

CITES - Convención Sobre el Comercio Internacional de Especies Amenazadas de Fauna y Flora Silvestres. 2013. Apéndices I, II y III de la, en vigor a partir del 12 de junio de 2013. http://www.cites.org/sites/default/files/ esp/app/2013/S-Appendices-2013-06-12.pdf. Fecha de consulta: 12 de enero de 2014.

CORMACARENA - Corporación para el desarrollo sostenible del área de manejo especial La Macarena. 2010. Comunicación interna P.M.G.A 3.10.1565. Respuesta a solicitud 007710 de julio 19 de 2010. Asunto: solicitud información flamingo. Villavicencio, Colombia.

Espinoza F, Parra L, Aranguren J, Martino A, Quijada M, Pirela $D$, Rivero $R$, Gutiérrez $T$, Jiménez $N$, Leal $S$, León E. Numbers and distribution of the Caribbean Flamingo in Venezuela. Waterbirds, 2000; 23: 80-86.
Fierro K. 2009. Aves Migratorias en Colombia.En: Amaya, JD, Naranjo LG (Editores) Plan Nacional de las Especies Migratorias: Diagnóstico e identificación de acciones para la conservación y el manejo sostenible de las especies migratorias de la biodiversidad en Colombia. MAVDT - WWF. Bogotá, Colombia, p. 6375, 193-204.

Hilty S, Brown W. 2001. Guía de las Aves de Colombia. Princenton University Press, Traducción al español por H. Álvarez (2001) American Bird Conservancy-ABC. p. 1030

Jiménez I, Cadena CD. Por qué no liberar animales silvestres decomisados. Ornitología Colombiana, 2004; 2: 53-57

Manchester SJ, Bullock JM. The impacts of non-native species on UK biodiversity and the effectiveness of control. Journal of Applied Ecology, J of Appl Ecol, 2000;37: 845-864.

Mancera NJ, Reyes O. Comercio de Fauna Silvestre en Colombia. Rev Fac Nal Agr Medellín, 2008; 61(2): 46184645.

MMA - Ministerio del Medio Ambiente. 2002. Estrategia Nacional para la Prevención y el Control del Tráfico Ilegal de Especies Silvestres. Dirección General de Ecosistemas. Ministerio del Medio Ambiente, Bogotá, Colombia, p.

McNeely JA, Mooney HA, Neville LE, Schei P, Waage JK. (Editores) 2001. A Global Strategy on Invasive Alien Species. IUCN Gland, Switzerland, and Cambridge, UK, p. 50.

McNish T. 2007. Las aves de los Llanos Orientales de Colombia. M y B. Bogotá, Colombia, p. 302.

Mock KE, Latch EK, Rhodes OE Jr. Assessing Losses of Genetic Diversity Due to Translocation: Long-Term Case Histories in Merriam's turkey (Meleagris gallopavo merriami). Conservation Genetics, 2004; 5: 631-645

Rangel JO, Aguilar M. 1995. Una aproximación sobre la diversidad climática en las regiones naturales de Colombia.: En Rangel JO. (Compilador) Colombia Diversidad Biótica 1. Instituto de Ciencias Naturales. Universidad Nacional de Colombia, Colombia, Bogotá, p. 25-76.

Rangel JO. 1998. Flora orinoquense. En: Fajardo M, Dominguez C, Molano BJ, Rangel O, Defler T, Rodríguez JV, Cavelier de FI, Gómez AJ, Publio PH, Barona B, G, Mejia M, Romero ME, Díaz O, Galeano C, Pérez L. Colombia Orinoco. Fondo FEN Colombia, Bogotá, Pp. 103-133.

Renjifo L.M, Franco-Maya AM, Amaya-Espinel JD, Kattan GH, López-Lanús B, (Editores). 2002. Libro rojo de aves de Colombia. Serie Libros Rojos de Especies Amenazadas de Colombia. Instituto de Investigación de Recursos Biológicos Alexander van Humboldt y Ministerio del Medio Ambiente, Bogotá, Colombia.

Rodríguez D, Pantaleón A. 2012. Phoenicopterus ruber. En: Naranjo LG, Amaya JD, Eusse-González D, Cifuentes-Sarmiento Y. (Edts.). Guía de las Especies Migratorias de la Biodiversidad en Colombia. Aves. Vol. 1. Ministerio de Ambiente y Desarrollo Sostenible / WWF Colombia. Bogotá, Colombia, p. 78-80 
Roe D. 2008. Trading Nature. A report, with case studies, on the contribution of wildlife trade management to sustainable livelihoods and the Millennium Development Goals. TRAFFIC Internationaland WWF International. Cambridge, UK, p. 100.

Romero-Ruiz M, Galindo G, Otero J, Armenteras D. 2004. Ecosistemas de la cuenca del Orinoco Colombiano. Instituto de Investigaciones en Recursos Biológicos Alexander von Humboldt e Instituto Geográfico Agustin Codazzi e IGAC, Bogotá, Colombia, p. 189.

Rueda RA. (Editores). 1999. Tráfico de fauna silvestre en Colombia. Latin América Environmental Society, IUCN,
Biocolombia. Informe final proyecto No. LA-5123. Bogotá, Colombia, p.144.

Ruiz- Guerra CJ, Johnston-González R, Cifuentes-Sarmiento Y, Estela FA, Castillo LF, Hernández CE, Naranjo LG. Noteworthy Bird Records from the Southern Chocó region of Colombia. Bull BrOrnClub, 2007; 127: 283-293.

Troncoso F. 2002. Phoenicopterus ruber. En: Renjifo LM, Franco AM, Amaya JD, Kattan G, López B. (eds). 2002. Libro Rojo de Aves de Colombia. Serie Libros rojos de especies amenazadas de Colombia. Instituto de Investigación de Recursos Biológicos Alexander Von Humboldt y Ministerio de Medio Ambiente, Bogotá, Colombia. p. 81. 